\title{
El Francés como segunda Lengua en la Formación de de Ingenieros Hispano-Parlantes: Un Debut
}

\author{
Lucio R. Cañete \\ Universidad de Santiago de Chile (USACH), Departamento de Ingeniería Industrial, \\ Lab. de Ingeniería Neurocognitiva. Av. Ecuador 3769, Estación Central, Santiago-Chile. \\ (e-mail: lucio.canete@usach.cl)
}

Recibido Jun. 07, 2012; Aceptado Jul. 13, 2012; Versión final recibida Sep. 12, 2012

\begin{abstract}
Resumen
Con el propósito de incrementar el capital intelectual de ingenieros hispano-parlantes se evalúan cuantitativamente alternativas para que el francés sea parte armónica y formal del proceso formativo de carreras tecnológicas en una universidad tradicional chilena. Puesto que no se disponía de información sobre los costos y beneficios que la inclusión de una segunda lengua extranjera provocaría en el desempeño profesional, se procedió a configurar diferentes diseños de la asignatura. Se encontró, a través de simulaciones y experimentos con voluntarios, que la mejor alternativa es enseñar francés una vez finalizado el primer año de la carrera a través de cuatro horas pedagógicas semanales, logrando así un dominio intermedio. Esta opción muestra que se incrementaría el ingreso promedio de los jóvenes titulados en más de 7\%, extendería su red comunicacional, ampliaría su acervo cultural y disminuiría discriminaciones por clase, entre otros impactos positivos.
\end{abstract}

\section{French as a Second Language in the Formation of Spanish-Speaking Engineers: A Premiere}

\begin{abstract}
With the purpose of increasing the intellectual capital of Spanish-speaking engineers, alternatives for teaching French are evaluated as harmonic and formal part of technological programs in a Chilean university. Since no information was available about the costs and benefits that the inclusion of a second foreign language would bring in their professional performances, different designs of the subject were analyzed. Through simulations and experiments with volunteers, it was found that the best alternative is to include French once the students finish the first year of their careers, with four hours per week, achieving an intermediate level. This option shows that the average income of young graduates would increase by more than $7 \%$, extending their communications network, expanding their cultural wealth and decreasing class discrimination, among other positive impacts.
\end{abstract}

Keywords: foreign language, engineer formation, French, intellectual capital 


\section{INTRODUCCION}

La Educación Superior tiene entre otros desafíos de actualidad, la formación de profesionales aptos para encarar los avances tecnológicos, la globalización y la inmersión en una sociedad del conocimiento. Así ciertas competencias que tradicionalmente se consideraban algo ajenas a las ingenierías, ahora son explícitamente declaras dentro de modelos educativos de varias universidades. Aceptando por una parte esta tendencia en la Universidad de Santiago de Chile (USACH) reflejada en su Modelo Educativo Institucional que señala el atributo de "Lenguaje, cultura e idioma" (VRA, 2007) y por otra parte confiando en diversas investigaciones catastradas por Garbin (2011) donde se evidencia que el manejo de una segunda lengua extranjera distinta al Inglés generaría notables impactos en el desempeño profesional ante situaciones problema; el presente proyecto formula y evalúa alternativas de asignaturas de Francés o Alemán para que sean incorporadas en forma obligatoria al proceso formativo de ingenieros.

Pese a que esta inquietud es antigua (Changeux, 1983); se ataca la incertidumbre de cuál de los dos idiomas sería el más útil, del nivel de dominio, de la cantidad de horas semanales, de la localización en la malla curricular y de la cantidad de semestres a cursar; como también de los recursos comprometidos (Bize et al., 1966). Ante ello el presente proyecto despliega diversas alternativas de asignaturas para los mencionados idiomas (Cañete y San Juan, 2010). También evalúa el desempeño de jóvenes profesionales con cierto dominio de estas lenguas no tan sólo en ámbitos puramente tecnológicos (Sunthonkanokpong, 2011), sino además en otras manifestaciones culturales (Andreou, 2005).

Como hipótesis se plantea entonces que la incorporación formal y explícita de una segunda lengua extranjera diferente al Inglés dentro del proceso formativo de ingenieros, genera en ellos cualidades diferenciadoras en los exigentes mercados actuales y futuros (De Los Ríos, 2010), redundando en un significativo valor agregado para estos profesionales y para la USACH, la universidad que los educó. En efecto, tal como lo expresa Díaz Barriga (2003), la búsqueda de mejoras al desempeño profesional de ingenieros y la disipación de la duda respecto al real aporte que para ello tendría el Francés o Alemán, se enmarcan dentro de la evaluación curricular, siendo el presente un problema de curriculum design o curriculum construction en una carrera formada por asignaturas.

El presente proyecto plantea entonces el siguiente Objetivo General: Formular y Evaluar la asignatura de Francés o Alemán en el proceso formativo de ingenieros. Este Objetivo General recién enunciado se puede descomponer en los siguientes Objetivos Específicos: 1) Precisión del Estado del Arte en materias de los beneficios y costos asociados a ambos idiomas en la formación de ingenieros, tanto para los alumnos como para la universidad. 2) Planteamiento grueso de diferentes alternativas de asignaturas obligatorias para ambos idiomas (nivel de dominio, cantidad de horas semanales, cantidad de semestres, obligatoria-voluntaria...). 3) Jerarquización de las alternativas de acuerdo a un criterio de rentabilidad. 4) Diseño en detalle de la alternativa más rentable.

Es importante insistir que la incorporación obligatoria del Alemán o Francés considerada en el presente proyecto, no excluye de modo alguno la incorporación del Inglés hasta lograr un profesional bilingüe en este último idioma. La incorporación del Inglés es una iniciativa complementaria al presente proyecto pero que se canalizará a través de otra vía, sin perjuicio de la interacción imprescindible entre ambas.

\section{METODOLOGÍA}

A continuación se describen las principales actividades realizadas desde mediados del año 2008 hasta finales del 2011 para alcanzar respectivamente cada uno de los cuatro objetivos específicos recién enunciados. Puesto que estos objetivos pretenden entregar información que permita una certera toma de decisiones, se ha preferido la evaluación propuesta por Stufflebeam y Shinkfield (1987) ya que ésta comprende contexto, insumos, proceso y productos; todos elementos íntimamente ligados en una evaluación curricular que como la presente busca medir y mejorar impactos. 
Para precisar la situación actual se recurre a literatura especializada en asuntos de idioma, se catastran reportes de universidades extranjeras y chilenas en temas afines y se examinan desde fuentes secundarias los estudios de mercado tanto de clientes como proveedores y competidores sobre profesionales que cuenten con el manejo de uno y de los dos idiomas en cuestión. Es decir, para alcanzar el primer objetivo específico se preferencia una evaluación curricular externa, extendida como la medición del rendimiento del egresado ante ciertas demandas del medio laboral.

Para el planteamiento grueso de las alternativas, a través de observación ecosistémica a fenómenos contemporáneos, se develan los impactos que en el desempeño profesional se pretenden. Posteriormente la actitud de los alumnos medida por medio de encuestas y la opinión técnica de los empleadores medida por encuestas y entrevistas en profundidad, orientan las distintas configuraciones (virtuales y heurísticas) de los recursos intelectuales y materiales (actuales y potenciales) de una unidad académica tecnológica dispuesta a someterse al presente proyecto, de tal forma de lograr distintas propuestas en cuanto a modalidades y contenidos de asignaturas.

Para jerarquizar las alternativas, primero se realiza una preselección cualitativa de acuerdo a las ventajas y desventajas utilizando la moda de ellas, para luego realizar un estudio prospectivo que cuantifica tanto los costos y beneficios que se generarán en los alumnos y luego en los futuros profesionales.

Finalmente para lograr el cuarto objetivo específico que es el diseño de la alternativa más rentable, se selecciona a docentes con experiencia en materias curriculares para que formulen el contenido de detalle de las unidades temáticas, elijan la bibliografía, configuren los laboratorios y perfilen los profesores que dictarán la asignatura.

\section{EL ESTADO ACTUAL}

Tal como se estableció en párrafos precedentes, el Modelo Educativo Institucional de la USACH establece nítidamente entre otros atributos al "Lenguaje, cultura e idiomas" como sello formativo. En efecto, diversos estudios demuestran que el desempeño profesional alcanza rendimientos superiores cuando desde etapas tempranas se tiene la posibilidad de interactuar en el Mercado de las Ideas (Fléaux et al., 2002). Lamentablemente, la formación en la Enseñanza Media, desde donde provienen los alumnos que ingresan a carreras tecnológicas de la USACH, adolece no tan sólo de una adecuada preparación en el manejo del Inglés como actual idioma universal; sino además y lo que es peor, de serias deficiencias en la expresión y comprensión por medio del Castellano como lengua madre. En el ámbito tecnológico los efectos negativos se evidencian cuando los estudiantes se privan de conocimientos de vanguardia y cuando una vez titulados no logran posiciones competitivas debido a deficiencias comunicacionales; donde la información es un elemento vital para la toma de decisiones (Cela-Conde y Ayala, 2007).

Ante este diagnóstico, el Departamento de Tecnologías Industriales de la USACH realizó en el 2008 con recursos propios un Estudio de Marginalidad Aditiva que confirma la precaria situación ya descrita. Este demostró que los jóvenes profesionales pueden incrementar sus rentas no tan sólo gracias al manejo del Inglés; sino además tal como se muestra la Figura 1, debido a cierto dominio de una segunda lengua extranjera: Francés o Alemán.

En efecto, los resultados de la mencionada investigación, demuestran tal como se aprecia en la barra de la derecha del gráfico de la Figura 1, que un incremento del manejo de una segunda lengua extranjera desde un nivel Bajo hasta Alto, repercutiría en \$95000 en la renta mensual de un Tecnólogo. Si se considera que cada joven profesional comienza a laborar a los 23 años de edad, se le provocaría según dicho estudio un costo oportunidad de casi \$ 48 millones hasta la fecha en que jubile. 


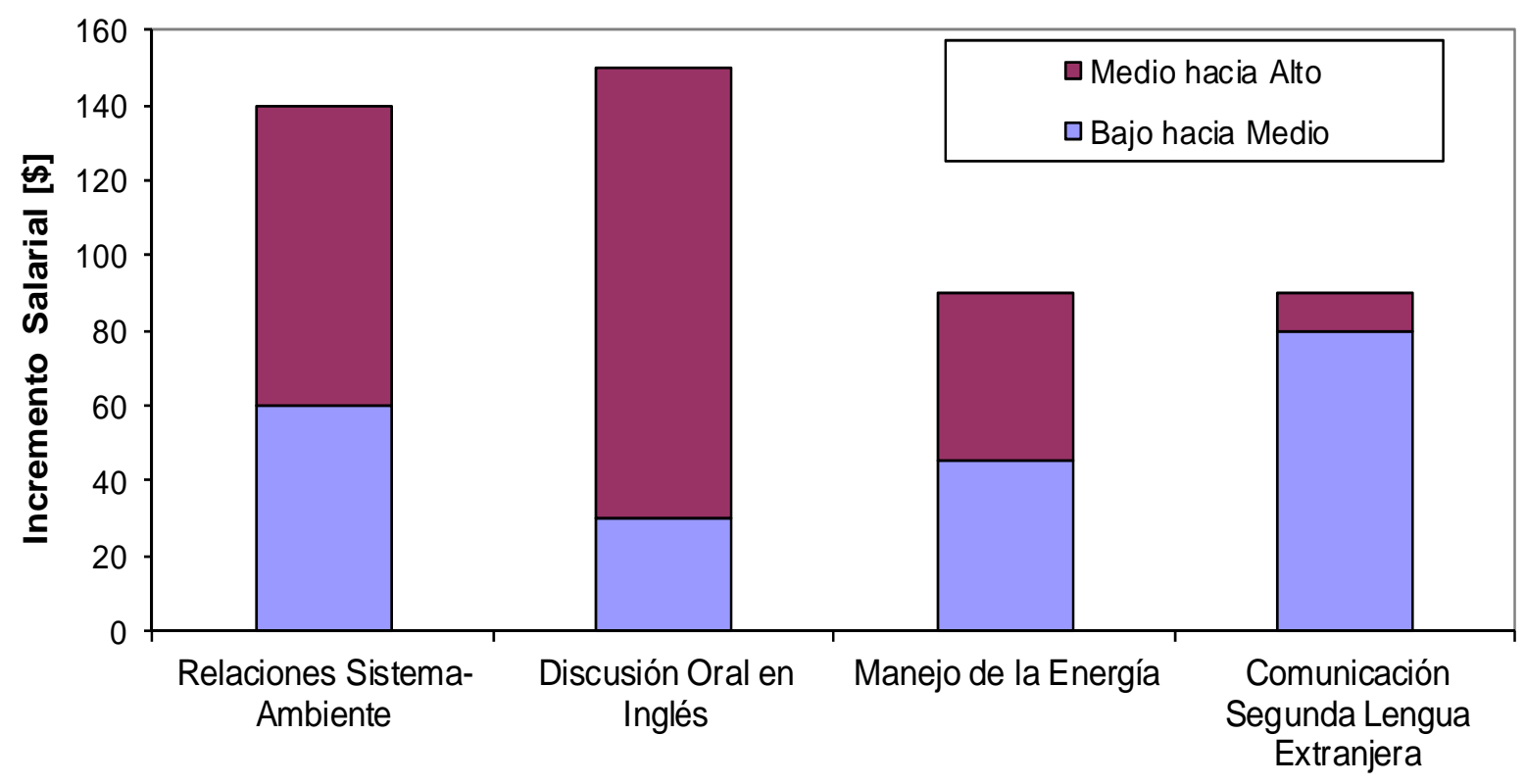

Competencia laboral

Fig. 1: Resultados del Estudio de Marginalidad Aditiva para impactos en la mejora formativa

Ciertamente este incremento llamó la atención por su magnitud y por ende mereció un cuestionamiento que se resolvió a través de una segunda ronda de preguntas hacia quienes ya habían manifestado la valoración acrecentada. Los argumentos dados en respuesta no versaron sobre asuntos exclusivos de las especialidades de ingenierías ni tecnologías propiamente tales; sino sobre aspectos conductuales tales como: a) la disciplina que se requiere para aprender una lengua extranjera, cualidad que se extrapola a otros ámbitos laborales, b) el afán de superación, fenómeno generalmente valorado en empresas competitivas y c) la curiosidad propia no tan sólo de científicos; sino de cualquier joven que emprende una carrera profesional.

Otro antecedente que refuerza la importancia de los idiomas al interior de la USACH (aunque no cuantitativo ni específico para el Francés y/o Alemán), se encuentra el 2010 en el informe "Actualización del Perfil de Egreso de la Especialidad de Telecomunicaciones" consignando ciertas habilidades "Instrumentales: relacionadas con habilidades cognitivas, metodológicas, tecnológicas y lingüísticas".

Por otra parte, considerando el Grado de Bachiller en Tecnología, el cual se obtiene en conjunto con el Título Profesional de Tecnólogo en Telecomunicaciones y que conduce sin mayores trámites a ingeniería, destaca el primer objetivo de dicho programa: "Proporcionar al estudiante una visión integral acerca del Hombre, la cultura y la sociedad, que permite a su vez la afirmación de su vocación de desarrollo personal". Esta declaración es completamente sinérgica con un programa de segunda lengua extranjera durante la formación profesional de un Tecnólogo por cuanto el manejo del Francés y/o Alemán constituye una vía para el desarrollo intelectual más allá de la adquisición de habilidades específicas tecnológicas.

Toda esta información proveniente específicamente de la carrera de Tecnólogo en Telecomunicaciones de la USACH (donde más del $85 \%$ de los egresados inmediatamente continúa estudiando ingeniería mientras laboran), fue crucial para comenzar a respaldar algunas hipótesis; manteniendo aún la incógnita respecto de la lengua a incluir en la formación profesional si de la evaluación así resultara. Sin embargo, la duda fue siempre entorno al Francés y Alemán, debido a que tal como establece Cristal (2003), obedecería a un asunto de entrar en sintonía con quienes ostentan un poder económico; que en este caso en particular se refleja en la industria, ciencia y tecnología. 


\section{Los argumentos para el Francés}

Aún cuando Chile no presenta inmigración francesa relevante que haya dejado su impronta e influido en gustos, ya el novelista Alberto Blest Gana en su best-seller Martín Rivas, identificaba a la francofilia o el gusto exacerbado por la cultura francesa en la sociedad chilena desde los albores de La Independencia. Al margen de dicha preferencia, que en rigor para fines académicos constituye una admiración y la búsqueda de un provecho a través de nuevo conocimiento, se identifican en la historia de Chile diversos hitos educativos. El más importante es sin duda la segunda lengua extranjera de carácter obligatorio que ya no existe en la Educación Media y que permitió a millones de chilenos que hoy superan los 46 años de edad, aprender una segunda lengua extranjera (mayoritariamente francés) desde $8^{\circ}$ Básico hasta $4^{\circ}$ Medio de la Educación Científico-Humanista. Sin duda, ahora algunos de ellos son padres de los jóvenes tecnólogos e ingenieros que actualmente se forman en la USACH y es de suponer que al interior de la familia tendrán una postura favorable respecto de una iniciativa como la que ahora se evalúa.

Además de la postura emocional que ineludiblemente influye en la toma de decisiones, existen ciertos argumentos concretos que invitan a enseñar Francés en carreras tecnológicas. La investigación más completa al respecto se presenta en un artículo de Richard Shryock del Virginia Polytechnic Institute and State University (Shryock, 2009), donde se mencionan las siguientes cualidades del idioma Francés: a) lengua oficial en 28 países de los 51 que forman parte de la Organisation Internationale de la Francophonie, b) idioma oficial junto al Inglés de otras organizaciones internacionales tales como: United Nations, UNESCO, NATO, Organization for Economic Cooperation and Development, International Labor Bureau, International Olympic Comité, European Community, Universal Postal Union, International Red Cross y Union of International Associations y c) idioma dominante en el trabajo de la European Court of Justice, del European Tribunal of First Instante y de la Press Room at the European Commission in Brussels.

En cuanto a ingeniería, la misma investigación señala los siguientes hitos para Francia como cuna y promotor del idioma en cuestión: a) país seleccionado por la comunidad científica internacional para el emplazamiento del primer reactor multinacional de fusión nuclear (International Thermonuclear Experimental Reactor), b) siete de sus "Top Ten" exportadores hacia Los Estados Unidos de Norteamérica pertenecen al rubro industrial de alta tecnología, c) cuarto productor mundial de automóviles (Renault, Peugeot, Citroën) y tercer exportador mundial automotriz, d) cuarto investigador dentro de la Organization for Economic Development and Cooperation (después de Japón, Alemania y Estados Unidos de Norteamérica), e) primer investigador mundial en energías, f) Líder mundial en investigaciones médicas (el virus del SIDA fue aislado por médicos franceses y el Human Genome Project está localizado en París), g) Líder en proyectos aeroespaciales (Aérospatiale, Arianespace, Airbus) y h) potencia militar mundial (después de Estados Unidos y Rusia) y segundo en el mundo en tecnología de defensa, destinando tropas bajo el Derecho Internacional en una decena de países que suman un contingente de 15000 soldados.

En Chile, están presentes más de una centena de empresas de capitales franceses, donde cuatro de ellas están exclusivamente en el rubro de las telecomunicaciones. También destaca la Cámara Franco Chilena para el Comercio y la Industria, la cual fue fundada en 1884 y cuenta en la actualidad con 230 empresas asociadas.

\section{Los argumentos para el Alemán}

Chile es uno de los pocos países en el mundo que registra una colonización alemana fomentada por el Estado. Quizás sea ese el principal vínculo con la cultura germana. En lo que respecta al interés manifestado por los estudiantes del Departamento de Tecnologías Industriales, en los sondeos previos afloraba espontáneamente "la disciplina" como el valor más frecuentemente mencionado y el desarrollo artefactual como la tecnología más admirada. 
En cuanto a la importancia del Alemán como lengua extranjera, las estadísticas del Department of Modern Languages de la University of Colorado Denver indican lo siguiente respecto al Alemán (Phillips, 2009): a) no sólo es la lengua oficial de Alemania y Austria; sino además se habla en Suiza, Liechtenstein, Luxembourg, norte de Italia, este de Bélgica y partes del este de Francia, b) más del $18 \%$ de los libros anualmente publicados en el mundo se escriben en Alemán, c) en algunos países europeos tales como Polonia y Hungría, es la lengua extranjera más recomendada por los científicos a sus alumnos, alcanzando el $70 \%$ de las preferencias, d) segunda lengua con más frecuencia utilizada en Internet y e) lengua materna de intelectuales influyentes tales como Goethe, Nietzsche, Kafka, Mozart, Bach, Beethoven, Freud y Einstein entre otros.

En cuanto a Alemania como cuna y promotor del idioma en cuestión, las estadísticas del ya citado Department of Modern Languages indican lo siguiente: a) más importante socio comercial de casi todos los países europeos y muchos otros países no europeos, b) segundo exportador mundial después de Estados Unidos de Norteamérica y c) tercera nación más industrializada del mundo.

Respecto a negocios tecnológicos, Alemania cuenta con más de 2500 subsidarias y afiliadas en Estados Unidos de Norteamérica. En Chile existen más de 80 empresas con capitales alemanes y destacando además la Cámara Chileno-Alemana de Comercio e Industria, la cual fue fundada en Valparaíso en 1916 y a la fecha actual posee 600 socios en Chile y en Alemania, siendo la cámara binacional europea más grande de Chile.

\section{PLANTEAMIENTO DE ALTERNATIVAS}

Aun cuando el resultado de las encuestas expuesto en la primera sección del presente artículo pueda en un comienzo sorprender, la duda se disipa al constatar la importancia mundial del idioma Francés y Alemán en la generación y transferencia de conocimiento desde la Revolución Industrial hasta el presente. Estos impactos no sólo se registran en las ciencias y tecnologías; sino además en la literatura, música, pintura y filosofía entre otras manifestaciones; es decir, en toda la cultura occidental (Henriot, 1946). Pues bien, estos mismos impactos son los que se pretende recrear a lo largo del proceso formativo de los estudiantes, particularmente en un mayor acceso a conocimiento tecnológico de especialidad, en la participación en el Mercado de las Ideas, ampliación de conexiones neuronales, en el incremento del capital intelectual y en una mejora de la disciplina. Por lo tanto, las alternativas a evaluar respecto de la segunda lengua extranjera deben imprescindiblemente pretender estos impactos que más adelante se detallan.

\section{1.- mayor acceso a conocimiento tecnológico de especialidad}

Por ahora los hispano-parlantes no son los líderes en la vanguardia tecnológica y los principales avances los generan y publican profesionales cuya lengua madre no es el Castellano. Pese a que la mayoría de esta tecnología se presenta en idioma Inglés, un porcentaje importante de ella es generada por francófonos y germano-parlantes. Por ejemplo: según el catálogo 2007 del Goethe Institut, el $40 \%$ de los investigadores norteamericanos recomienda a sus alumnos lecturas científicas en Alemán pues la consideran la segunda en importancia a nivel mundial.

\section{2.- participación en el Mercado de las Ideas}

En la actualidad según Eurostat (Oficina de Estadística de las Comunidades Europeas en Luxemburgo), más del $40 \%$ de la población de la Unión Europea habla Francés o Alemán, siendo este último el más popular de dicho mercado ( $\mathrm{y}$ no el Inglés como normalmente en Latinoamérica se cree). Así un estudiante chileno con el dominio de uno de estos dos idiomas puede comprender y ser comprendido por individuos de su misma especie que tienen otras formas diferentes de pensar. Esto no solo brindará acceso a conocimiento tecnológico especializado; sino que amplía la posibilidad de participar en eventos académicos de diversa índole.

\section{3.- ampliación de conexiones neuronales}


Está demostrado que el aprendizaje de un nuevo idioma por un joven no tan sólo facilita el aprendizaje de otros que se estudien con posterioridad; sino que afianza los precedentes. En tal contexto, se espera que un manejo del Alemán o Francés refuerce al Castellano y al precario Inglés, como también agilice la asimilación de idiomas que el futuro profesional en su etapa laboral desee conocer.

\section{4.- incremento del capital intelectual}

Los alumnos que generalmente ingresan a una especialidad de Tecnólogo en el Departamento de Tecnologías Industriales de la USACH, poseen un historial de privaciones económicas, sintiéndose en frecuentes ocasiones disminuidos y/o tratados en forma peyorativa en diversos contextos sociales. En tal sentido, el dominio de una segunda lengua extranjera permitirá un incremento del capital intelectual de los jóvenes estudiantes, aumentando la valoración de ellos por parte de la sociedad y en tal sentido, integrar el selecto grupo de chilenos que dominan más de un idioma extranjero.

\section{5.- mejora disciplinaria}

Con frecuencia los profesores se quejan de la falta de cumplimiento de lo demandado como la puntualidad en tareas, del escaso orden en los informes escritos y de la poca observancia a cuestiones reglamentarias mínimas por parte de la generalidad de los alumnos de la carreras tecnológicas. En tal sentido, el aprendizaje de una segunda lengua extranjera sin duda requiere de una conducta algo preterida en la actualidad: disciplina. Los motivos de la ausencia de un comportamiento disciplinado en cuanto a responsabilidades de estudiante universitario escapan a los objetivos del presente proyecto; pero sí se puede afirmar que asumir el Alemán o el Francés como lengua de estudio obliga a cualquier joven a disciplinarse, particularmente cuando enfrenta la gramática. Por ello se espera que tal conducta demandada para aprobar la segunda lengua extranjera, se presente en las otras múltiples actividades de la vida del alumno.

\section{La actitud de los alumnos}

Imprescindible también resulta conocer la actitud de los alumnos ante las alternativas de aprender una segunda lengua extranjera durante su proceso formativo, más aún cuando tal iniciativa es pionera en Chile en carreras tecnológicas y afines. Para ello se diseñó y aplicó una encuesta a finales del segundo semestre del 2008 a 46 estudiantes que estaban cursando o habían cursado la asignatura de Formulación y Evaluación de Proyectos Tecnológicos y que por tanto, se supone, tenían cierta madurez para responder un cuestionario con propósitos de mejora continua en su educación.

El cuestionario constó de sólo cuatro preguntas más los requerimientos de los datos del encuestado, incluyendo la pregunta si deseaba que la información entregada por él fuese divulgada. Este instrumento tuvo una amplia acogida y he aquí la respuesta graficada en la Figura 2 a la primera interrogante de cual idioma prefería aprender.

De la figura 2 se desprende que casi existe un empate en las preferencias totales de los alumnos entre Francés y Alemán; y que además, cualquiera de ellos por sí solo supera a la suma de los demás (Italiano, Portugués y otros). Sin embargo, la más atractiva de ambas lenguas es el Alemán, mencionándose en primera preferencia 24 veces, superando a las primeras preferencias del Francés que alcanzan 15 menciones. También se observó que los alumnos provenientes de Educación Científico - Humanista tienen una inclinación más por el Francés, mientras que los encuestados provenientes de la Educación Técnico - Profesional se inclinan más por el Alemán. En todo caso, la totalidad de los encuestados mencionó una de las dos lenguas en cuestión, ya sea en primera, segunda o tercera preferencia de todas las alternativas disponibles. Esta reacción fue un incentivo para continuar con la evaluación del presente proyecto 


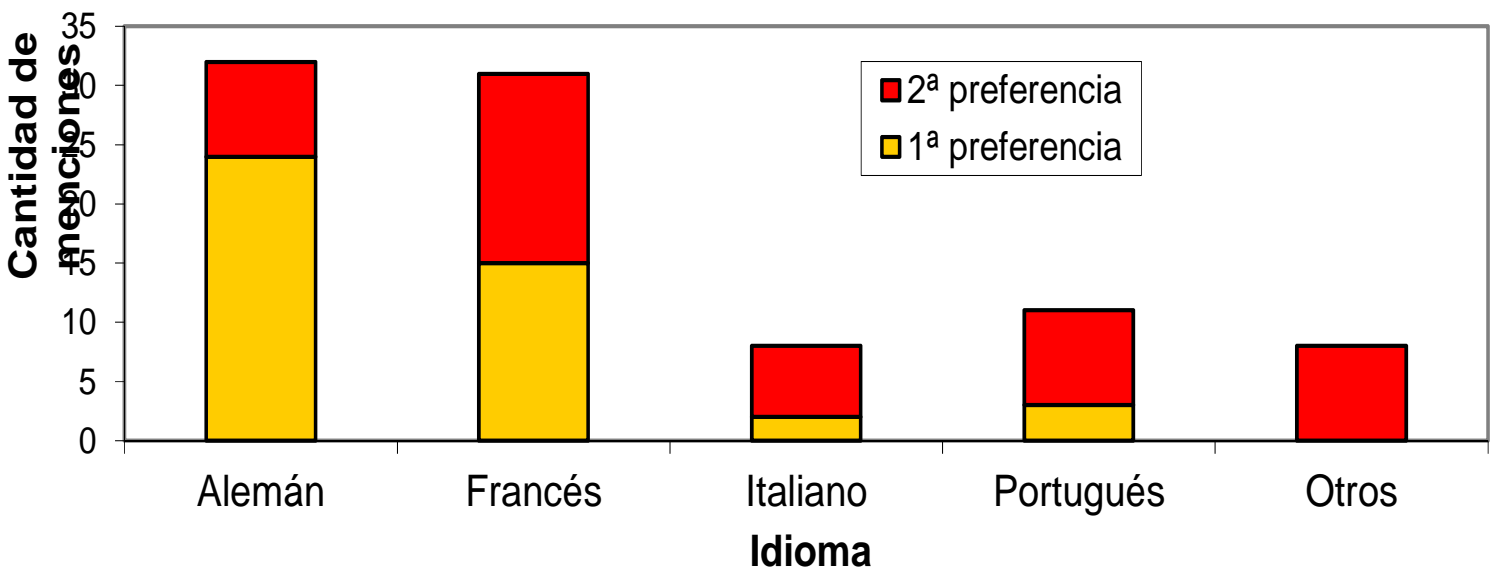

Fig. 2: Preferencias de los alumnos para diferentes idiomas

Otro asunto de interés es estimar el sacrificio que cada estudiante estaría dispuesto a realizar por aprender una segunda lengua extranjera. Esta duda se disipó al responder una segunda pregunta que arrojó que sólo el 3\% estaba dispuesto a entregarle menos de 2 horas cronológicas de estudio personal a la semana.

Por otra parte, de acuerdo al Modelo Educativo Institucional, la formación profesional en la USACH debe ser integral y al respecto interesa conocer el provecho que en diferentes actividades humanas puede provocar el manejo del Francés o Alemán. En tal sentido, se formuló una tercera pregunta cuyas respuestas se grafican en la Figura 3. Del gráfico se desprende que el mayor provecho se obtendría a través del acceso a la web, corroborando las prácticas recreativas de la generación actual en orden a obtener y entregar información en temas diversos con carácter de entretención. Otro asunto a notar es aquel denominado "Símbolo de estatus", donde se obtuvo una respuesta con una frecuencia del $15 \%$, asunto que se discutirá más adelante en el presente informe.

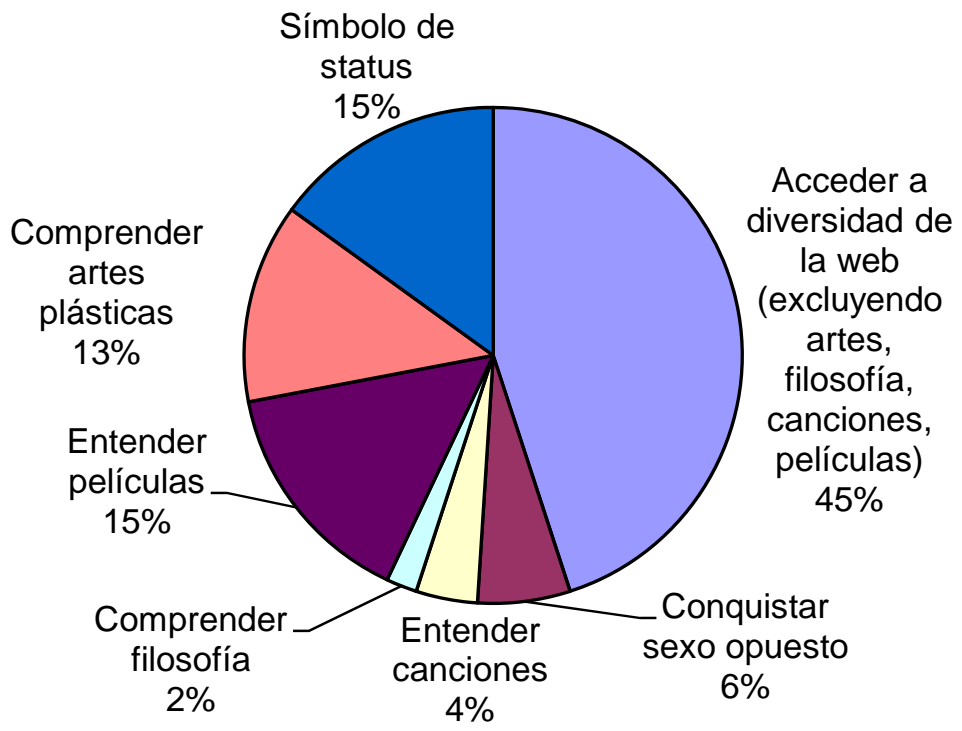

Fig. 3: Pronósticos de los alumnos sobre su provecho de una segunda lengua extranjera

Finalmente, para conocer el pronóstico respecto al impacto en ámbitos laborales que el manejo de una lengua le generaría, se formuló una cuarta pregunta para un escenario ambicioso cuya respuesta develó que más del $85 \%$ espera poder laborar en Europa gracias al manejo de la segunda lengua extranjera. 


\section{¿ Obligatoria o voluntaria?}

En forma espontánea emerge la interrogante de que los alumnos decidan si cursan una lengua extranjera o si tal ruta debe ser imprescindible para titularse. Ante tal disyuntiva, la alternativa de voluntaria tiene un par de desventajas serias. La primera es que el Francés o Alemán no constituirían en tal caso un sello formativo de la USACH, siendo una cualidad portada por sólo aquellos que hubiesen preferido cursar las asignaturas ofrecidas. La otra desventaja es la más grave y se sustenta en la experiencia, la cual indica que una actividad voluntaria en las generaciones actuales tiene muy pocos efectos en los alumnos por la ausencia de constancia en ellos. En efecto, al comenzar el año 2009 a los estudiantes que habían aprobado la asignatura de Formulación y Evaluación de Proyectos Tecnológicos se les ofreció la posibilidad de cursar el seminario experimental en Francés "Technologies et Possibilisme", el cual al inicio tuvo una gran acogida con una veintena de inscritos. Sin embargo, al cabo de dos meses los asistentes regulares no superaron la media docena, pese a los estímulos existentes. Una experiencia similar se repitió el año 2011, notándose la misma deserción. Por lo tanto, el carácter de voluntario de la asignatura irá en desmedro de la calidad corporativa de ésta, diluyéndose el valor agregado que se pretende en todos los titulados.

\section{¿Cuál nivel de dominio?}

Hasta ahora se ha planteado la necesidad de que los titulados de la carrera al momento de enfrentarse al medio laboral manejen el Francés o Alemán; sin embargo, nada se ha propuesto específicamente respecto del nivel de dicho dominio. Sin embargo, desde el Estado del Arte del presente informe ya pueden deducirse explícitamente ciertas habilidades de expresión (Productive Skills) y comprensión (Receptive Skills) entre las cuales destacan: naturalidad, abstracción, fluidez y complejidad. Estas cualidades pueden ser buscadas en algún marco universal de dominio como el de la Association of Language Testers of Europe (ALTE), encontrándose que según dicha referencia, el nivel ideal de manejo para fines profesionales y culturales establecidos en el presente proyecto correspondería al B2 y en un caso más desfavorable al B1. La experiencia indica que para alcanzar el nivel B2 en un escenario universitario hispano-parlante se necesitan aproximadamente 380 horas de clases para el Francés, mientras que para el Alemán se requieren 420 horas de clases. Por lo tanto si ambas asignaturas ocupan la misma cantidad de horas totales de clases, la lengua germana demandará de más dedicación, tanto de parte del alumno como del profesor.

\section{¿Cuántos semestres y cuantas horas semanales?}

Una vez que el alumno selecciona la segunda lengua extranjera que en forma obligatoria deberá cursar, emerge la duda respecto a cuantos semestres durará el programa. En tal sentido se debe tener presente que el nivel que pretende darse no es el básico, por el contrario es aquel que permita al titulado defender sin mucha dificultad una idea. También debe considerarse el aspecto de intensidad; es decir, la cantidad de horas de clases a la semana.

Resumiendo en función de la cantidad de horas totales se presentan al menos cuatro fórmulas. Una consiste en dictar las asignaturas durante el segundo y tercer año; es decir, constaría de cuatro semestres con 4 horas pedagógicas a la semana (considerando como hora pedagógica a 45 minutos de clase alumno frente a profesor). La segunda fórmula también ocupa cuatro semestres pero considera 6 horas pedagógicas semanales. Las otras dos fórmulas también consideran 4 o 6 horas de clases, pero las asignaturas comenzarían en el primer año, extendiéndose hasta el segundo y tercero. Es importante observar que la fórmula menos exigente en cuanto a carga académica, aquella de cuatro semestres con 4 horas de clases semanales e igual cantidad de horas de estudio personal, se logra al final del programa con un total de 272 horas; cantidad inferior a las 380 horas de clase necesarias para alcanzar el nivel B2. Es decir, se podría llegar sólo al nivel B1, cuyas habilidades no asegurarían todos los impactos deseados. Sin embargo, el nivel B1 aunque no siendo el ideal al momento de titulación, configurado con herramientas de auto-aprendizaje facilitará el ascenso hacia el nivel B2 y superiores. 
Otro aspecto que merece observarse es aquel donde los 4 semestres para la alternativa que permite un nivel B1, se localizan al final de la carrera de tecnólogo; es decir, durante el $2^{\circ}$ y $3^{\circ}$ año. Esta ubicación temporal tiene también desventajas y ventajas. La principal desventaja es que el aprendizaje comienza con un año de retraso y por ende los beneficios también se desfasan. La ventaja es que el alumno tiene un año más de madurez para seleccionar la lengua preferida, elección irreversible.

\section{JERARQUIZACION DE ALTERNATIVAS}

\section{Incorporando novedad}

De acuerdo a lo discutido en el presente proyecto, quedan tres alternativas de interés real para ser evaluadas. La primera consiste en lograr un profesional con un nivel semi-avanzado de dominio, el cual se denominará como B2 de acuerdo a la terminología ALTE. La segunda consiste en lograr un profesional con un nivel intermedio de dominio, denominado B1 según la misma terminología. Finalmente la tercera alternativa consiste en no incluir la segunda lengua extranjera en forma obligatoria, denominada como alternativa CERO, es decir, aquella que corresponde a la situación base

Es importante mencionar que las alternativas B1 y B2 se evalúan frente a una malla curricular como la actual, la cual está formada por 36 asignaturas y respecto de ellas no se retira ninguna en reemplazo de la Segunda Lengua Extranjera que se incorpora. Es decir, no se trata de una Novedad Sustitutiva; sino de una Novedad Aditiva por cuanto corresponde a un elemento nuevo que se agrega al sistema productivo sin sacar otro (Toffler, 1985). Dicho elemento nuevo es según la elección del alumno, el Francés o el Alemán. Entonces, si se supone una malla curricular como la vigente, ésta incrementará en un 8\% la cantidad de horas totales con la alternativa B1 y en un $12 \%$ con la alternativa B2.

Esta nueva malla curricular ampliada en una asignatura en cuatro semestres si se trata de la alternativa B1, o en todos los semestres si se trata de la alternativa B2, no luce sobrecargada si se considera que se trata de la especialidad de Tecnólogo en Telecomunicaciones donde los fenómenos de mensajes, códigos y lenguajes son pertinentes a la formación profesional. Si no es posible en el corto plazo modificar la Malla Curricular; pueden usarse como medida transitoria las asignaturas co-programáticas, pero éstas debido a la cantidad total de horas, no permitirían alcanzar un nivel B1.

\section{Cuantificación de costos y beneficios}

Evidentemente, la iniciativa de una segunda lengua extranjera es un proyecto y como tal debe evaluarse según las herramientas que la disciplina de la Ingeniería Económica dispone para ello (Newnan et al., 2004). Ante ésto emerge la primera interrogante respecto de cual agente económico se hará la evaluación: ¿ el profesional ?, ¿ la USACH ?, ¿ la sociedad ?. Puesto que el objetivo del Departamento de Tecnologías Industriales es entregar a la sociedad profesionales exitosos, la evaluación del proyecto se hará para un titulado tipo. Esto, sin perjuicio de identificar y contabilizar los ingresos y egresos que al Departamento de Tecnologías Industriales como unidad responsable del proceso formativo se le generarán.

Otra cuestión es respecto de la longitud del horizonte de planeación; es decir, del lapso de tiempo durante el cual el proyecto será evaluado. Ante ello, el proyecto será evaluado para los tres años que dura la carrera y para los tres años siguientes una vez titulado. Tan sólo se consideran tres años de titulación pues un tiempo más prolongado implicaría realizar pronósticos de variables naturales y culturales de difícil predicción con el estado de la técnica actual ante escenarios tan dinámicos como los que hoy se observan.

De acuerdo a conversaciones en profundidad con los alumnos lideradas por los expertos en el presente proyecto, éstos disponen en general de bastantes horas de ocio sin destinación bien definida, las cuales oscilan entre las 6 y 8 horas semanales. En tal sentido, la asignatura puede 
ocupar ese tiempo con la salvedad de que el curso de lengua extranjera debe ser considerado por los alumnos con un claro componente recreativo. Si esto ocurre, el costo oportunidad por sacrificar actividades de ocio regulares puede despreciarse. Otro costo relevante para los alumnos son aquellos destinados al material de estudio, el cual puede estimarse en $\$ 25000$ por semestre. Los beneficios comienzan a ser percibidos una vez avanzado el primer semestre de la asignatura en curso, los cuales son de naturaleza hedónica. También se incluyen el aumento en el rendimiento en los trabajos de investigación, los cuales según estimaciones pueden estimarse en ahorros para generar trabajos de investigación, los cuales promedian $\$ 60000$ por cada semestre de avance en la asignatura.

Sin embargo, los ingresos más relevantes se logran una vez que se pierde la calidad de alumno y se obtiene la de titulado, donde tal como el Estudio de Marginalidad Aditiva anteriormente citado lo consigna, el mercado premia en unidades monetarias un manejo de la segunda lengua extranjera. Tal recompensa sería de alrededor de \$ 80000 mensuales si el nivel de manejo es Medio y de \$ 90000 si el manejo es Alto. Asumiendo un probable sobredimensionamiento de tales cifras y en una postura conservadora, se considerará un promedio de $\$ 70000$ mensual o $\$ 420000$ semestral para la alternativa B1 y de $\$ 80000$ o de $\$ 480000$ semestrales para B2.

Se desprende entonces que la alternativa B2 supera ligeramente a la B1, y ambas son superiores a la alternativa CERO (no enseñar segunda lengua extranjera). Asumiendo una tasa de interés semestral del 5\%, se tiene que tanto B1 como B2 entregan un Valor Actualizado Neto bastante similar para los tres años de estudiantes más los primeros tres de titulados; los cuales considerando la precisión y exactitud de los datos de entrada, no permiten una discriminación más fina debido a los supuestos del modelo. En lo que no cabe duda alguna, es que cualquiera de las dos alternativas que incluye la segunda lengua extranjera, supera a la alternativa CERO.

\section{FORMULACION DEL FRANCÉS Y SUS RESULTADOS}

\section{Disponibilidad de recursos institucionales}

El nivel de precisión del presente estudio no permite desempatar las preferencias asociadas al Francés respecto del Alemán como tampoco respecto de los impactos que en forma diferenciada cada uno de ellos provocará en los jóvenes titulados.

Sin embargo, atendiendo a la disponibilidad de recursos docentes por parte de la universidad sí es posible seleccionar una de las dos lenguas ante la restricción que los resultados del estudio sólo dan cabida a una de ellas. Así considerando que la cantidad de profesores capacitados en el francés triplica al Alemán, se ha decido por esta lengua romance. Se insiste en que ésta es una opción provisoria y que responde a una limitante de factores productivos de tipo coyuntural, dejando completamente abierta la posibilidad de que cada alumno opte por el Alemán cuando existan presupuestos institucionales más holgados.

\section{Configuración final y experimentos complementarios}

Tal como se deduce de los párrafos precedentes, los impactos deseados se logran cuando el idealmente el estudiante logra un nivel de manejo B2, el cual según los patrones internacionales se refiere a que la persona es capaz de entender las ideas principales de textos complejos que traten de temas tanto concretos como abstractos, incluso si son de carácter técnico, siempre que estén dentro de su campo de especialización. Dicha persona puede relacionarse con hablantes nativos con un grado suficiente de fluidez y naturalidad, de modo que la comunicación se realiza sin esfuerzo por parte de los interlocutores. Puede además producir textos claros y detallados sobre temas diversos. Estos impactos se observaron en las pruebas piloto en 25 voluntarios, notándose que trascurrido un año de aprendizaje, ellos eran capaces de enriquecer sus trabajos de especialidad con bibliografía técnica en Francés.

Para alcanzar este nivel, los docentes prepararon entretenidas pedagogías usando las herramientas multimediales, notando que durante los dos semestres de prueba piloto, los alumnos asumían la asignatura como una actividad recreativa. De hecho la parte que marcó menos inasistencia y la que demandó por parte de los alumnos más tareas para la casa ainiciativa de 
ellos mismos, fue aquella de cantar canciones (generalmente lentas) de clásicos en francés. Así tuvo una amplia acogida la lectura y canto simultáneo de canciones tales como "Michele" interpretada por Gérard Lemonam en el caso de los varones y "Avant de nous dire adieu" interpretada por Jeane Manson en el caso de las mujeres.

Una vez configurada la opción para el Francés al nivel B2 y ante la legítima duda de su real funcionamiento pese a todas las proyecciones realizadas, se procedió a explicar la iniciativa a diferentes profesionales ajenos al desarrollo del proyecto, pidiéndoles su libre opinión; como también a realizar testeos complementarios.

Un tema que afloró fue aquello que ciertamente según lo demuestra un histórico artículo científico, donde se demuestra que el mercado laboral chileno tiene un fuerte efecto discriminatorio no tanto por sexo, edad, estudios de postgrado, calidad académica escolar ni otras variables de control estándar; sino por clase (Núñez y Gutiérrez, 2004). En tal sentido, la opinión de 35 encuestados encargados de seleccionar personal de una muestra de 64, afirmó que una persona que visiblemente maneja Francés, tiene en Chile poca probabilidad de ser discriminado por clase. Otra información interesante se obtuvo al exponer a cada una de 27 personas una docena de CVs muy similares entre sí, diferenciándose conspicuamente en que la mitad de ellos declaraba dominio del Francés y la otra no. El grupo de CVs que declaró el dominio de la lengua gala superó en un $32 \%$ la calificación total neta del otro.

Finalmente se identificó a 8 Ingenieros Civiles en Geografía y 5 Ingenieras Civiles en Industrias que estudiaron por cuenta propia el Francés, quienes afirmaron que en sus respectivas organizaciones por el sólo hecho de dominar dicha lengua, ascendieron puestos que repercutieron en incrementos salariales del 7\%. También afirmaron que durante su etapa laboral ya titulados, para ello jamás tuvieron que leer o hablar en Francés, ocupando dicho idioma sólo en asuntos personales extra-laborales.

Otros resultados cuantitativos del presente trabajo se muestran en la Figura 4 donde se compara la trayectoria de un joven en su etapa de estudiante de ingeniería civil (6 años de duración de la carrera de pregrado) y en su temprana etapa laboral como titulado (4 primeros años de egresado). En ella se aprecia que dedicarse a la asignatura de Francés implicaría al comienzo unos costos que castigan la utilidad neta en su quehacer de estudiante, pero que a partir del cuarto de la carrera universitaria, ésta comienzaría a crecer. La diferencia se aprecia nítidamente una vez titulado, donde la curva del rendimiento con manejo de Francés, se distancia por sobre de la curva del rendimiento sin esta segunda lengua.

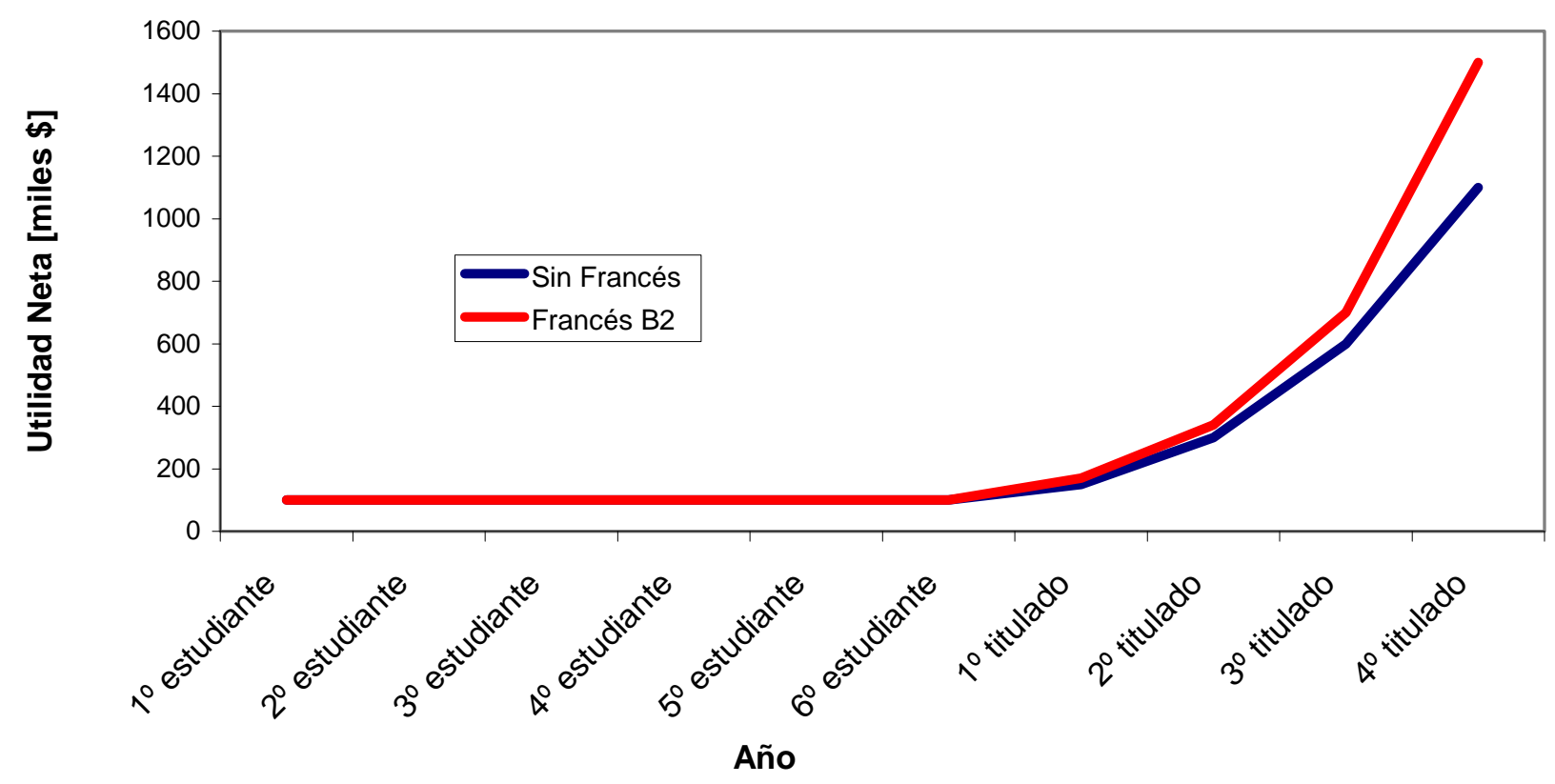

Fig. 4: Simulación comparada del ingreso neto para ingenieros civiles 


\section{CONCLUSIONES}

La inquietud abordada en el presente trabajo se enmarca dentro de la evaluación curricular y aun cuando ésta es una actividad normal, no lo son los cuestionamientos respecto de mejoras en el proceso formativo de ingenieros hispano-parlantes a través de una segunda lengua extranjera. En efecto, las imprescindibles evaluaciones periódicas se hacen preferentemente para actualizaciones curriculares en temas tecnológicos y aquellas en temas humanistas y afines, no consideran el desempeño que tendrían los ingenieros dotados de cierto dominio de una segunda lengua extranjera adquirido de manera obligatoria en la formación de pregrado.

Las alternativas para configurar la lengua extranjera en cuestión se desplegaron en busca de impactos en diversas áreas de la cultura, persiguiendo mejoras en el desempeño de profesionales no sólo en ámbitos puramente tecnológicos; sino que en diversas facetas de sus quehaceres como personas. Los experimentos realizados se facilitaron en parte posiblemente por el reconocimiento de la globalización como un hecho cierto y por otra parte por la novedad que implica transitar por un escenario laboral altamente competitivo con una herramienta distinta.

La inclusión de Francés demostró que podría incrementar el salario, que enriquece el capital intelectual y que aumentaría la inmunidad ante la discriminación laboral. También el Alemán ofrece ventajas similares, tan sólo que por ciertas restricciones presupuestarias dicha lengua no fue monitoreada en el presente proyecto, pero a la luz de los resultados de la investigación se pronostica que los impactos del idioma germano también sean positivos y en los mismos ámbitos.

Puesto que la configuración de la asignatura de Francés fue concebida utilizando técnicas pedagógicas modernas combinadas estratégicamente con juegos, los sacrificios por parte de los estudiantes son mínimos, considerando el eficiente aprendizaje como una entretenida aventura. Al respecto es importante comentar que las mismas tecnologías de información y comunicación que los jóvenes aprenden en sus clases regulares de asignaturas tecnológicas, sirvieron para facilitar el aprendizaje del Francés a través de medios multimediales y similares. Por lo que es de suponer que conforme más se desarrollen dichas tecnologías, más expedito será el aprendizaje de la segunda lengua extranjera por parte de los tecnólogos.

Pese a que la lengua extranjera fue adicionada a la malla curricular sin retirar alguna otra asignatura en su reemplazo, la formación tradicional en materias propiamente tecnológicas no se afectó; mostrando en algunos casos, efectos sinérgicos con ellas. Sin embargo el Francés no mostró provechos utilitarios notorios en la comunicación oral en dicha lengua propiamente tal; sino en otras actividades que se relacionan con una ampliación de la cosmovisión.

¿ Qué viene ahora ? Ciertamente el presente trabajo es un proyecto de innovación docente y como tal merece seguir avanzando a etapas más desarrolladas las cuales se alimentarán con información de otras precedentes. En lo inmediato resulta útil ampliar la representatividad de las muestras de estudiantes sometidos a experimentación e idealmente, realizar un plan piloto de un par de semestres de duración para luego hacerle el seguimiento a los titulados y cuantificar en ambientes laborales los impactos.

Aun cuando el presente estudio correspondió a la enseñanza de una segunda lengua extranjera en la formación de ingenieros cuya formación de pregrado es de seis años de duración impartida por una universidad pública chilena, la problemática expuesta puede tener bastantes elementos comunes con otras carreras y universidades hispanoamericanas. Incluso el seguimiento señalado en el párrafo anterior se potenciaría si dicho plan es llevado a cabo en paralelo con otras universidades hispanoparlantes. Por ello se espera que el estudio y sus resultados sean aplicables, con los respectivos ajustes, en otros escenarios.

\section{AGRADECIMIENTOS}

El autor expresa sus agradecimientos a la Vicerrectoría Académica de la USACH, en particular a su Programa de Concursos de Innovación Docente que permitió financiar el presente proyecto y a 
su Unidad de Innovación Educativa que está apoyando la continuidad de la investigación respecto a la Segunda Lengua Extranjera.

\section{REFERENCIAS}

Andreou, E., G. Andreou y F. Vlachos, Studying orientations and performance on verbal fluency tasks in a second language, Learning and Individual Differences: (15)1, 23-33, (2005).

Bize, P.R., P. Goguellin y R. Carpentier, Le Penser Efficace, $1^{\text {a }}$ edición, SEDES, Paris, 78-97, (1966).

Cañete, L. y E. San Juan, Formulación y Evaluación de la Asignatura Obligatoria Segunda Lengua Extranjera (Francés o Alemán) en la Formación Profesional del Tecnólogo en Telecomunicaciones, Actas de la $15^{\circ}$ Convención Científica de Ingeniería y Arquitectura, 338-356, La Habana, Cuba, 29 de Noviembre al 3 de Diciembre (2010).

Díaz Barriga, Á., Currículum. Tensiones conceptuales y prácticas, Revista Electrónica de Investigación Educativa, ISSN: 1607-4041 (en línea): 5 (2), 2003. http://redie.ens.uabc.mx/vol5no2/contenidodiazbarriga.html. Acceso de Marzo (2012).

Cela-Conde, C. y F. Ayala, Human Evolution: trails from the past, $1^{a}$ edición, Oxford University Press, Nueva York, 339-340, (2007).

Changeux, J-P., L' Homme Neuronal, 2ª edición, Fayard, Paris, 56-58, (1983).

Crystal, D., English as a Global Languaje, 2ª edición, Cambridge University Press, Cambridge, 7, (2003).

De Los Ríos, I., A. Cazorla, J. Díaz-Puente y J. Yagüe, Project-Based Learning in Engineering Higher education: two decades of teaching competences in real environments, Procedia - Social and Behavioral Sciences: (2)2, 1368-1378, (2010).

Fléaux, R., B. Armaud y P. Jean-Baptiste, L'Homme de Trop, Sciences et Avenir: (23)1, 34-35, (2002).

Garbin, G. y otros seis autores, Neural Bases of Language switching in High and Early Proficient Bilinguals, Brian and Language: (119)4, 119-121, (2011).

Henriot, E., La France Immortale, $1^{\text {a }}$ edición, Librairie Hachette, Paris, 104-108, (1946).

Newnan, D., T.G. Eschenbach y J.P. Lavelle, Engineering Economic Analysis, 9a edición, Oxford University Press, Nueva York, 4-15, (2004).

Nuñez, J. y R. Gutierrez, Class Discrimination and Meritocracy in the Labor Market: evidence form Chile, Estudios de Economía: (31)2, 113-132, (2004).

Phillips, T., The importance of German (en línea), 2009, http://carbon.cudenver.edu/\%7Etphillip/importantgerman.html Acceso: 4 mayo 2010.

Stufflebeam, D. y A. Shinkfield, Evaluación Sistemática: guía teórica y práctica, 1ª edición, Paidos-MEC, Barcelona, 23-57, (1987).

Shryock, R., French, The Most Practical Foreign Language, (en línea), 2008, http://www.fll.vt.edu/French/whyfrench.html Acceso: 4 junio 2012

Sunthonkanokpong, W., Future Global Visions of Engineering Education, Procedia Engineering, (8)1, 160164, (2011).

Toffler A., The Adaptive Corporation, $2^{\circ}$ edición, McGraw-Hill, Nueva York, 68-69, (1985).

VRA: Vicerrectoría Académica de la Universidad de Santiago de Chile, Modelo Educativo Institucional, $1^{\text {a }}$ edición, Imprenta USACH, 25-26, (2007). 